\title{
Quantification and identification of sperm subpopulations using computer-aided sperm analysis and species-specific cut-off values for swimming speed
}

\section{Liana Maree and Gerhard van der Horst}

\begin{abstract}
Motility is an essential characteristic of all flagellated spermatozoa and assessment of this parameter is one criterion for most semen or sperm evaluations. Computer-aided sperm analysis (CASA) can be used to measure sperm motility more objectively and accurately than manual methods, provided that analysis techniques are standardized. Previous studies have shown that evaluation of sperm subpopulations is more important than analyzing the total motile sperm population alone. We developed a quantitative method to determine cut-off values for swimming speed to identify three sperm subpopulations. We used the Sperm Class Analyzer ${ }^{\circledR}$ (SCA) CASA system to assess the total percentage of motile spermatozoa in a sperm preparation as well as the percentages of rapid, medium and slow swimming spermatozoa for six mammalian species. Curvilinear velocity (VCL) cut-off values were adjusted manually for each species to include 80\% rapid, $15 \%$ medium and 5\% slow swimming spermatozoa. Our results indicate that the same VCL intervals cannot be used for all species to classify spermatozoa according to swimming speed. After VCL intervals were adjusted for each species, three unique sperm subpopulations could be identified. The effects of medical treatments on sperm motility become apparent in changes in the distribution of spermatozoa among the three swimming speed classes.
\end{abstract}

Motility is an important characteristic of spermatozoa, especially flagellated spermatozoa. Because the ultimate goal of a spermatozoon is to fertilize an ovum, it must be motile and travel relatively long distances in internal fertilizers (Holt and van Look 2004). This type of motility is characteristic of freshly ejaculated mammalian spermatozoa, where the flagellum generates a symmetrical and low amplitude waveform that propels the spermatozoon in a relatively straight line (Turner 2003).

The importance of sperm motility is highlighted further by the fact that measurement of this parameter is an essential criterion of most semen or sperm evaluations (Mocé and Graham 2008). One frequently encountered problem in subfertile or infertile males is a low population of motile spermatozoa (asthenozoospermia) (Mortimer 1994). Earlier studies have demonstrated a relationship between decreased sperm motility and decreased fertilization rates, which often requires resorting to assisted reproductive techniques to achieve fertilization (Turner 2003). Greater sperm motility also has been 
related to increased fertilization rates in vitro and pregnancy success (Zinaman et al. 2000, Sifer et al. 2005). In addition, the percentage of morphologically normal sperm and the size of some, but not all, sperm components (e.g., head length and total sperm length) have been correlated positively with the percentage of motile spermatozoa and sperm swimming speed (Malo et al. 2005, Gomendio et al. 2007, Humphries et al. 2008).

The standard method for evaluating sperm motility has been, and still is, manual scoring of the percentage of motile spermatozoa in a semen sample. This relatively subjective method, however, has been subject to large inter-laboratory variation (Jørgensen et al. 1997). The introduction of computer-aided sperm analysis (CASA) systems has led to more objective and sophisticated motility assessments (Agarwal et al. 2003, Mocé and Graham 2008). The CASA technique has the advantage of increasing the accuracy and reproducibility of measurements of sperm concentration and percentage of motile sperm (Rijsselaere et al. 2005). Furthermore, CASA permits determination of various sperm movement characteristics (kinematics) and the detection of subtle changes in sperm motion that cannot be identified by conventional manual sperm motility analysis (Abaigar et al. 2001, Agarwal et al. 2003, Rijsselaere et al. 2005).

Many investigators have used CASA to demonstrate the existence of sperm subpopulations in various mammalian species including pig, gazelle, horse, red deer, dog and rabbit (Abaigar et al. 1999, 2001, Quintero-Moreno et al. 2003, 2004, 2007, Martinez-Pastor et al. 2005, Núñez-Martínez et al. 2006). It now is recommended that sperm subpopulations be evaluated rather than relying of mean values for the entire sperm population, because mean values for motility oversimplify the analysis and decrease the usefulness of the data (Abaigar et al. 1999, Martinez-Pastor et al. 2005). Overall mean values for sperm motility also can mask the effects of drug treatment on spermatozoa, especially if certain subpopulations are more responsive to the treatment in species with heterogeneous ejaculates (Núñez-Martínez et al. 2006). Because assessment of sperm subpopulations has been linked to the fertility potential of the ejaculate, studying subpopulations should be considered for improving the sensitivity of semen analysis (Quintero-Moreno et al. 2003, NúñezMartínez et al. 2006).

To increase the potential value of CASA, it is imperative that all capturing techniques and analytical methods are standardized. This allows comparison of CASA-generated data among research laboratories and with previously published studies. The practical application of CASA, therefore, requires consideration of factors including temperature dependency of spermatozoa, effect of preparation (chamber) depth, duration of observations, number of trajectories required for reliable analysis, kinematic thresholds of progressive spermatozoa, and assessment of different motility parameters (Mortimer 1994, Kraemer et al. 1998, Holt and van Look 2004).

We investigated the cut-off values for sperm swimming speed to identify three subpopulations of spermatozoa. We present here a standardized, quantitative 
technique using CASA technology to assess the quality of sperm motility in different mammalian species.

\section{Materials and methods \\ Species studied}

We studied six mammalian species including human (Homo sapiens), chacma baboon (Papio ursinus), rhesus monkey (Macaca mulatta), vervet monkey (Chlorocebus aethiops), merino ram (Ovis orientalis) and house mouse (Mus musculus). Ethical approval was obtained from the Ethics Committee of the University of the Western Cape. The Helsinki Declaration governed research on humans (Christie 2000) and each human donor gave written consent. Husbandry and collection details for each species are presented in Table 1 and have been described previously as indicated.

\section{Collection and evaluation of semen/sperm samples}

Human semen samples were obtained by masturbation after two to three days of sexual abstinence. Semen samples from non-human primate species (chacma baboon, rhesus monkey, vervet monkey) and merino rams were obtained by rectal probe electroejaculation. Semen evaluation included measurement of volume, sperm concentration, percentage motility and osmolality as described previously (van der Horst and Maree 2009). The average semen parameters are given in Table 2. Mouse spermatozoa were collected by removal of the reproductive tract and dissection of the cauda epididymis (Tayama et al. 2006). Semen or spermatozoa were incubated for $5^{-60} \mathrm{~min}$ at $37 \square \square \mathrm{C}$ in a $\mathrm{CO}_{2}$ incubator before further analysis. Ten semen/sperm samples were selected for each species based on the quality of the samples.

\section{Selection of motile spermatozoa}

Ham's F10 medium (Invitrogen, Cape Town, South Africa), supplemented with 3\% bovine serum albumin (BSA) (Sigma, Cape Town, South Africa), was the preferred medium for our study, because it sustains sperm functions of various species for long periods (Brinders 1994, Mahadevan et al. 1997).

\begin{tabular}{|c|c|c|c|}
\hline Common name & Species & Husbandry details & Collection unit \\
\hline Human & Homo sapiens & $\begin{array}{l}\text { Donor program (Maree et al. } \\
\text { 2010) }\end{array}$ & $\begin{array}{l}\text { Dept. Biomedical Sciences, } \\
\text { Stellenbosch University }\end{array}$ \\
\hline Chacma baboon & Papio ursinus & $\begin{array}{l}\text { Housed outdoors (de Villiers and } \\
\text { Seier 2010) }\end{array}$ & $\begin{array}{l}\text { Delft Animal Centre, Medical Research } \\
\text { Council, Cape Town }\end{array}$ \\
\hline Rhesus monkey & Macaca mulatta & $\begin{array}{l}\text { Housed indoors (Parsons et al. } \\
\text { 2010) }\end{array}$ & $\begin{array}{l}\text { Delft Animal Centre, Medical Research } \\
\text { Council, Cape Town }\end{array}$ \\
\hline Vervet monkey & $\begin{array}{l}\text { Chlorocebus } \\
\text { aethiops }\end{array}$ & Housed indoors (Seier 1986) & $\begin{array}{l}\text { Primate Unit, Medical Research } \\
\text { Council, Cape Town }\end{array}$ \\
\hline Merino sheep & Ovis orientalis & $\begin{array}{l}\text { Housed outdoors (Cloete et al. } \\
\text { 2004) }\end{array}$ & $\begin{array}{l}\text { Elsenburg Experimental Farm, } \\
\text { Elsenburg Agricultural Centre }\end{array}$ \\
\hline Mouse (C57 strain) & Mus musculus & $\begin{array}{l}\text { Animal room, standardized } \\
\text { conditions }\end{array}$ & $\begin{array}{l}\text { Dept. Medical Biosciences, University } \\
\text { of the Western Cape }\end{array}$ \\
\hline
\end{tabular}

Motile spermatozoa from human, nonhuman primates and merino ram were selected using a swim-up technique described by van der Horst and Maree (2009). Motile spermatozoa from the mouse were selected by a swim-out technique. 
A small piece of mouse cauda epididymis was placed in a pre-warmed petri dish containing Ham 's F10 medium. After motile spermatozoa swam out of the duct and into the surrounding medium, a sample was taken from the edge of the spermatozoal cloud within 5 min for verification of sperm motility. The final concentration and total motility percentage of spermatozoa in the selected samples are given in Table 2.

\section{CASA equipment, capturing properties and analysis}

Sperm motility in semen and sperm preparations was assessed using the Motility/Concentration module of the Sperm Class Analyzer ${ }^{\circledR}$ (SCA) version 4.0.0.5 or 4.1.0.1 (Microptic S.L., Barcelona, Spain). Capturing data were collected using a Basler A602fc digital camera (Microptic S.L.) that was mounted (C-mount) on either a Nikon Eclipse 50i microscope (IMP, Cape Town, South Africa) or an Olympus CH2 microscope (Wirsam, Cape Town, South Africa), both equipped with phase contrast optics and a heated stage.

The capturing properties of the $\mathrm{SCA}^{\circledR}$ system were set as follows: number of images, 50; images/ sec, 50 or 75; optics, Ph- (negative phase contrast); chamber, Leja 20; and automatic analysis. The analytical properties for the various mammalian species are given in Table 3.

Table 2. Semen characteristics and motile sperm preparations of selected mammalian species (10 individuals for each species)

\begin{tabular}{|c|c|c|c|c|c|c|}
\hline & HS & PU & RM & CA & O० & MM \\
\hline \multicolumn{7}{|l|}{ Semen characteristics } \\
\hline Volume (ml) & $3.5 \pm 0.8$ & $0.9 \pm 0.5$ & $0.4 \pm 0.2$ & $0.7 \pm 0.3$ & $1.0 \pm 0.4$ & - \\
\hline $\begin{array}{l}\text { Sperm concentration } \\
\left(\times 10^{6} / \mathrm{ml}\right)\end{array}$ & $63.9 \pm 18.6$ & $212.5 \pm 252.6$ & $193.6 \pm 122.9$ & $72.4 \pm 18.0$ & $3846.1 \pm 2772.9$ & - \\
\hline Motility (\%) & $72.8 \pm 15.9$ & $73.4 \pm 16.7$ & $69.2 \pm 10.2$ & $57.4 \pm 15.5$ & $74.1 \pm 10.7$ & - \\
\hline Osmolality (mOsm/kg) & $356.8 \pm 19.8$ & $332.3 \pm 9.6$ & $352.5 \pm 15.8$ & $340.1 \pm 17.6$ & ND & - \\
\hline \multicolumn{7}{|c|}{ Motile sperm preparations } \\
\hline Semen $(\mu \mathrm{l})$ & $200-300$ & $50-100$ & 50 & $50-100$ & $10-40$ & - \\
\hline Ham's F10 $(\mu \mathrm{l})$ & $500-600$ & 150 & $100-150$ & 150 & 1000 & - \\
\hline $\begin{array}{l}\text { Sperm concentration } \\
\quad\left(\times 10^{6} / \mathrm{ml}\right)\end{array}$ & $3.2 \pm 1.1$ & $2.4 \pm 1.4$ & $6.4 \pm 2.7$ & $5.9 \pm 5.7$ & $6.6 \pm 4.2$ & $1.7 \pm 0.8$ \\
\hline Motility (\%) & $94.5 \pm 3.2$ & $93.9 \pm 2.9$ & $94.0 \pm 5.5$ & $94.7 \pm 4.7$ & $84.6 \pm 5.6$ & $89.7 \pm 6.2$ \\
\hline
\end{tabular}

Mean \pm SD

HS, Homo sapiens; PU, Papio ursinus (chacma baboon); RM, Macaca mulatta (rhesus monkey); CA, Chlorocebus aethiops (vervet monkey); OO, Ovis orientalis (merino ram); MM, Mus musculus (house mouse); ND, not determined.

Particle area was determined by visually evaluating captured fields and selecting the particle area size range that resulted in detecting only the sperm head for motility analysis, thereby excluding any other cells or debris. Curvilinear velocity (VCL) intervals for the three classes of sperm swimming speed were selected arbitrarily as the default values for initial motility analysis. Selection of default and adjusted motility analysis properties are discussed further below. The $\mathrm{SCA}^{\circledR}$ default values were used, however, for progressiveness and circularity of sperm tracks for all six species. Connectivity was set by visually evaluating captured fields and selecting the setting where individual sperm tracks were captured as continuous tracks. The number of average path velocity (VAP) points was selected by visual interpolation of what the average path would look like if it were drawn manually onto the curvilinear path of individual spermatozoa. This method 
avoided selecting a number of points that were too high or too low to be used for the smoothing of the average path (Mortimer 1997).

\section{Standardized procedure and parameters for sperm motility analysis}

Motility parameters were assessed at 10 and 30 min after a motile sperm population was prepared by either the swim-up or swim-out method. Each sperm population was evaluated by pipetting $5 \mu \mathrm{l}$ of preparation into a $20 \mu \mathrm{m}$ deep chamber of a pre-warmed $\left(37^{\circ} \mathrm{C}\right)$ Leja slide (Leja Products B.V., Nieuw-Vennep, The Netherlands). Depending on sperm concentration, two to ten fields were captured with the $\mathrm{SCA}^{\circledR}$ system until a total of 200 motile spermatozoa were analyzed as recommended by Mortimer (1994) and the World Health Organization (1999). Because sperm motility is sensitive to changes in temperature, the slide was removed from the slide holder and re-heated on the microscope stage for approximately $20 \mathrm{sec}$ between capturing events. Fields were captured randomly to eliminate bias toward higher sperm concentration or motility, but fields that included debris or clumps of sperm were avoided to decrease faulty analysis. All captured sperm tracks were verified visually to delete incorrectly recorded tracks, e.g., due to colliding spermatozoa.

The SCA ${ }^{\circledR}$ system automatically detects the sperm head (visualized as a white dot with phase contrast optics) and makes rapid and accurate measurements of various sperm motility parameters. Eight parameters were assessed including the curvilinear velocity (VCL), straight line velocity (VSL), average path velocity (VAP), linearity (LIN), straightness (STR), wobble (WOB), amplitude of lateral head displacement (ALH) and beat cross frequency (BCF) (van der Horst et al. 2011). The ALH parameter was measured as half the width of the VCL track rather the full VCL wave or double the riser values (risers method) as described by Mortimer (1994, 1997).

\section{Adaptation of $S C A^{\circledR}$ to identify sperm subpopulations}

The SCA ${ }^{\circledR}$ system provides various options for measurements of sperm motility to be used for evaluation of a semen/sperm sample, e.g., individual kinematic parameters; percentage total motility; progressive motility; rapid, medium and slow swimming spermatozoa; and type a-d classification as recommended by the World Health Organization (1999).

Table 3. SCA ${ }^{\circledR}$ motility analysis properties for six mammalian species investigated

\begin{tabular}{lllllll}
\hline & \multicolumn{1}{c}{ HS } & \multicolumn{1}{c}{ PU } & \multicolumn{1}{c}{ RM } & \multicolumn{1}{c}{ CA } & \multicolumn{1}{c}{ OO } & MM \\
\hline Species* & Human & Human & Human & Human & Ram & Rodent \\
Particle area $\left(\mu \mathrm{m}^{2}\right)$ & $20<\mathrm{PA}<80$ & $20<\mathrm{PA}<80$ & $20<\mathrm{PA}<80$ & $10<\mathrm{PA}<90$ & $15<\mathrm{PA}<70$ & $20<\mathrm{PA}<70$ \\
VCL Slow $(\mu \mathrm{m} / \mathrm{s})$ & $20<\mathrm{S}<30$ & $50<\mathrm{S}<80$ & $50<\mathrm{S}<80$ & $50<\mathrm{S}<80$ & $50<\mathrm{S}<80$ & $50<\mathrm{S}<80$ \\
VCL Medium $(\mu \mathrm{m} / \mathrm{s})$ & $30<\mathrm{M}<50$ & $80<\mathrm{M}<120$ & $80<\mathrm{M}<120$ & $80<\mathrm{M}<120$ & $80<\mathrm{M}<120$ & $80<\mathrm{M}<120$ \\
VCL Rapid $(\mu \mathrm{m} / \mathrm{s})$ & $\mathrm{R}>50$ & $\mathrm{R}>120$ & $\mathrm{R}>120$ & $\mathrm{R}>120$ & $\mathrm{R}>120$ & $\mathrm{R}>120$ \\
Progressivity $(\% \mathrm{STR})$ & $>80$ & $>80$ & $>80$ & $>80$ & $>80$ & $>80$ \\
Circular $(\%$ of LIN) & $<50$ & $<50$ & $<50$ & $<50$ & $<50$ & $<50$ \\
Connectivity & 12 & 20 & 20 & 12 & $20-25$ & 40 \\
VAP points $50 \mathrm{f} / \mathrm{s}$ & 7 & 7 & 7 & 7 & 7 & 11 \\
VAP points 75 f/s & 9 & 11 & 9 & 11 & 11 & 15 \\
\hline
\end{tabular}

HS, Homo sapiens; PU, Papio ursinus (chacma baboon); RM, Macaca mulatta (rhesus monkey); CA, Chlorocebus aethiops (vervet monkey); OO, Ovis orientalis (merino ram); MM, Mus musculus (house mouse).

*Species name selected from list of options from $\mathrm{SCA}^{\circledR}$ system.

VCL, curvilinear velocity; STR, straightness; LIN, linearity; VAP, average path velocity; f/s, frames per second; PA, particle area; S, slow; $\mathrm{M}$, medium; R, rapid. 
All of these measurements are presented here as an average of the total number of captured fields in the $\mathrm{SCA}^{\circledR}$ system's motility reports.

We investigated further the measurement of rapid, medium and slow swimming spermatozoa. Our method for measuring sperm motility used VCL intervals as cut-off points to classify spermatozoa into three swimming speed classes. We were particularly interested in determining the proper VCL cut-off values that should be selected for each species for future studies and the kinematic parameters of the three subpopulations compared to those of the total motile sperm population.

The percentage total motile spermatozoa and the rapid, medium and slow swimming spermatozoa in sperm preparations were evaluated first by selecting the same default VCL intervals for most species (Table 3). We selected these default values initially as preliminary cut-off values and for use as a standard method for sperm motility analysis. We selected these default values mainly for two reasons: 1) the default values of the SCA ${ }^{\circledR}$ system were set to evaluate human spermatozoa at 25 frames/sec, thus they are not entirely applicable to other species, and 2) to compare the motility characteristics of spermatozoa of various species. Only human spermatozoa were analyzed using lower default VCL intervals for the three swimming speed classes (Table 3), because the swimming speed for human sperm is much slower than the other species evaluated.

The VCL intervals for swimming speed classes of all six species were adjusted manually to include approximately $80 \%$ rapid swimming spermatozoa, $15 \%$ medium swimming spermatozoa and 5\% slow swimming spermatozoa. These percentages were calculated from the percentage of total motile spermatozoa, e.g., if a sample had 90\% total motility, the VCL intervals were adjusted eventually to include $72 \%$ rapid swimming spermatozoa, $13.5 \%$ medium swimming spermatozoa and $4.5 \%$ slow swimming spermatozoa. The cut-off percentages of spermatozoa in each swimming speed class (80, 15 and 5\%) for each species were selected arbitrarily as representing a good quality motile sperm sample.

\section{Statistical analysis}

MedCalc ${ }^{\circledR}$ version 10.4.0.0 (Mariakerke, Gent, Belgium) was used for basic statistical analysis. Tests were performed for normality of distribution and most data sets were characterized by normal distributions. Levene's test for equality of variances was applied and when $p>0.05$, one way analysis of variance analysis (ANOVA) was performed for parametric data distributions. Any significant differences $(p<0.05)$ indicated in the ANOVA table between groups were analyzed further using the Student-Newman-Keuls test for pairwise comparisons. If Levene's test gave $p<0.05$, independent T-tests with equal or unequal variances (depending on F-test result) were employed for individual differences. In subsets of data that appeared to have nonparametric data distributions, the Kruskal-Wallis test was employed and elaborated further for individual differences using the Mann-Whitney test for independent samples. Data are represented as mean \pm standard deviation (SD) in the tables and $p<0.05$ was considered significant. 


\section{Results}

The overall average, default and adjusted sperm motility and kinematic parameters of six mammalian species, captured at 50 frames/sec and 5-10 min after the sperm preparation was made, are presented in Table 4. The total percentage of motile spermatozoa (Total Average) is the sum of the percentage of motile spermatozoa in the rapid, medium and slow swimming speed classes (Rapid Default + Medium Default + Slow Default or Rapid Adjusted + Medium Adjusted + Slow Adjusted). The percentages of motile spermatozoa in the "default" swimming speed classes (Rapid, Medium, Slow Default) were determined by the default values selected for VCL intervals (Table 3) according to the standard method of sperm motility comparisons used in our laboratory. Among the six species evaluated, only the chacma baboon and merino ram had a significantly lower percentage of motile spermatozoa in the Rapid Default category compared to Total Average ( $p<0.05$ ); however, Rapid Default still comprised more than $90 \%$ of the total motile spermatozoa in these two species.

The change in the percentages of motile spermatozoa in the "adjusted" swimming speed classes (Rapid, Medium, and Slow Adjusted) was due to adaption of the VCL intervals to $80 \%$ rapid, $15 \%$ medium and 5\% slow swimming spermatozoa. A summary of the VCL cut-off values for the default and adjusted sperm swimming speed classes of each species at different time intervals and frame rates are given in Table 5. The VCL intervals for the adjusted swimming speed classes indicate that these intervals were unique for each species (no statistical comparison was done). No significant intraspecific differences in the VCL cut-off values were found between the two frame rates or two time intervals (Table 5). 


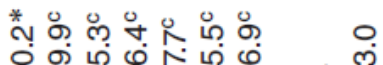
$+1+1+1+1+1+1+1$ l $1+1$ ச

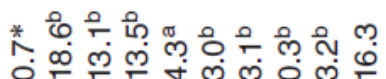
$+1+1+1+1+1+1+1+1+1+1$

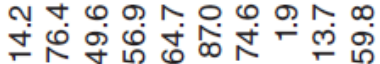

* ง

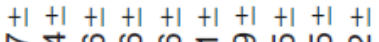
ผें

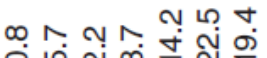

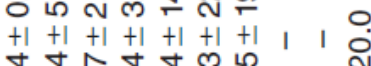

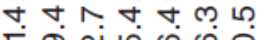
늉ํㅇ

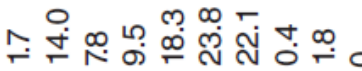
$+1+1+1+1+1+1+1+1+100$ ง ผูं

究

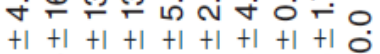

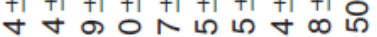

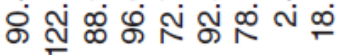

న็ $m$ 스 $+1+1+1+1+1+1+1+1+1$

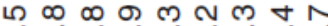
க்

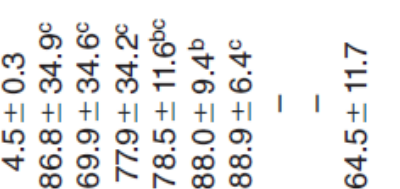

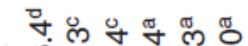

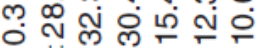
$+1+1+1+1+1+1+1 \quad 1$ $0 r+t m-0$ मुं

ㅇํㅇำำำ

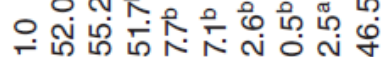

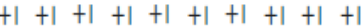
- 0 O N T

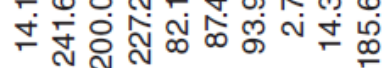

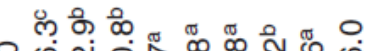
운

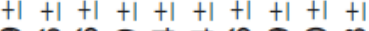
户

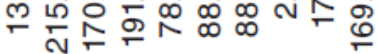

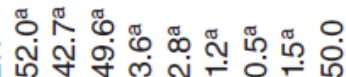
i in m N N L

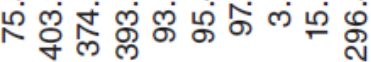

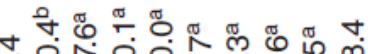
+ จิ

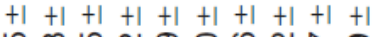

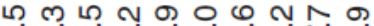
ค่

ก. ก T

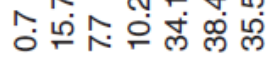

品

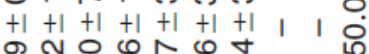

○

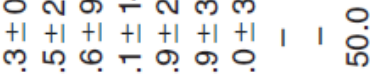

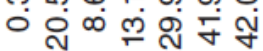

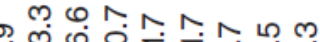
○ ल

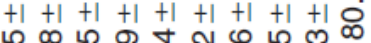

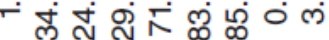

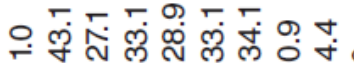
06 in 0 U $\infty \infty \infty$ ம்

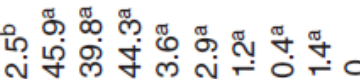

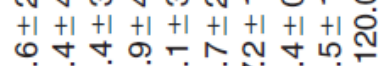

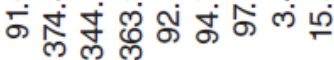

พัฒ

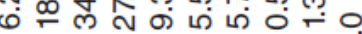
$+1+1+1+1+1+1+1+1+100$ 이둔 ภู่

ฐ

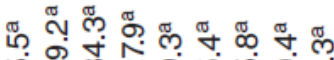
$+1+1+1+1+1+1+1+1+1$ and nonntz

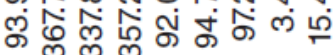




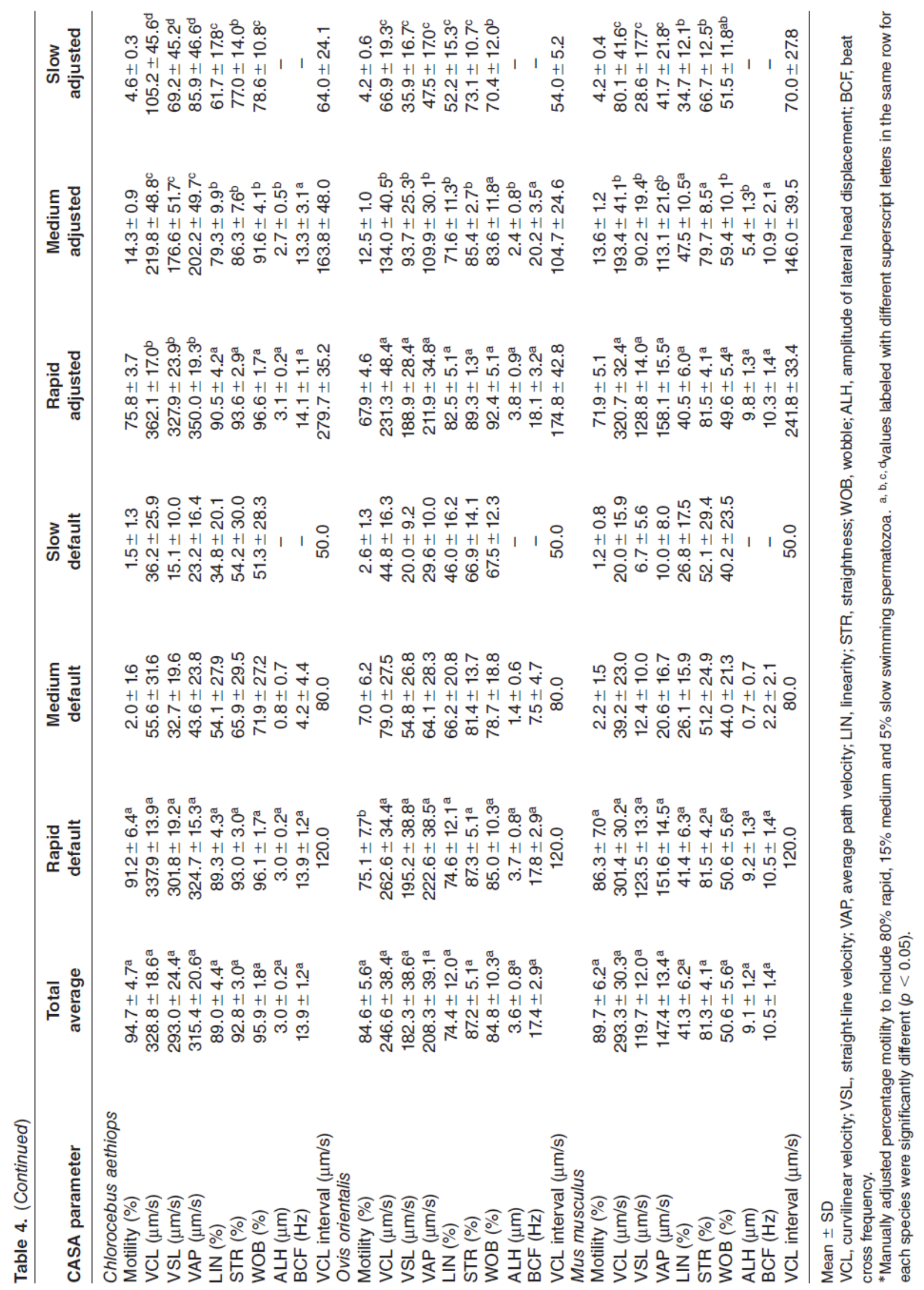


Table 5. Average default and adjusted VCL interval cut-off values for the six mammalian species when sperm motility was captured at two different frame rates and time intervals by the $\mathrm{SCA}^{\circledR}$ system

\begin{tabular}{lcrrrrrr}
\hline Swimming speed class & Capturing properties & HS & PU & RM & CA & OO & MM \\
\hline Rapid Default & & 50.0 & 120.0 & 120.0 & 120.0 & 120.0 & 120.0 \\
Rapid Adjusted & $10 \mathrm{~min}, 50 \mathrm{f} / \mathrm{s}$ & 89.2 & 296.3 & 239.9 & 279.7 & 174.8 & 241.8 \\
& $30 \mathrm{~min}, 50 \mathrm{f} / \mathrm{s}$ & 82.9 & 275.5 & 242.5 & 254.0 & 158.3 & 211.3 \\
& $10 \mathrm{~min}, 75 \mathrm{f} / \mathrm{s}$ & 101.8 & 315.2 & 265.4 & 295.5 & 183.8 & 283.4 \\
& & 89.1 & 281.4 & 285.6 & 287.5 & 154.2 & 237.2 \\
Medium Default & & 30.0 & 80.0 & 80.0 & 80.0 & 80.0 & 80.0 \\
Medium Adjusted & $10 \mathrm{~min}, 50 \mathrm{f} / \mathrm{s}$ & 59.8 & 185.6 & 169.8 & 163.8 & 104.7 & 146.0 \\
& $30 \mathrm{~min}, 50 \mathrm{f} / \mathrm{s}$ & 48.3 & 157.7 & 160.3 & 128.0 & 88.7 & 143.5 \\
& $10 \mathrm{~min}, 75 \mathrm{f} / \mathrm{s}$ & 69.3 & 202.1 & 191.0 & 185.7 & 78.6 & 169.1 \\
& $30 \mathrm{~min}, 75 \mathrm{f} / \mathrm{s}$ & 56.0 & 176.6 & 163.3 & 163.7 & 78.8 & 120.0 \\
Slow Default & & 20.0 & 50.0 & 50.0 & 50.0 & 50.0 & 50.0 \\
Slow Adjusted & $10 \mathrm{~min}, 50 \mathrm{f} / \mathrm{s}$ & 22.8 & 64.5 & 81.0 & 64.0 & 54.0 & 70.0 \\
& $30 \mathrm{~min}, 50 \mathrm{f} / \mathrm{s}$ & 22.4 & 59.5 & 69.5 & 56.5 & 51.0 & 62.5 \\
& $10 \mathrm{~min}, 75 \mathrm{f} / \mathrm{s}$ & 24.7 & 48.0 & 70.5 & 75.0 & 39.6 & 52.9 \\
& $30 \mathrm{~min}, 75 \mathrm{f} / \mathrm{s}$ & 26.0 & 69.8 & 68.6 & 50.6 & 46.5 & 51.6 \\
\hline
\end{tabular}

HS, Homo sapiens (human); PU, Papio ursinus (chacma baboon); RM, Macaca mulatta (rhesus monkey); CA, Chlorocebus aethiops (vervet monkey); OO, Ovis orientalis (merino ram); MM, Mus musculus (house mouse).

VCL, curvilinear velocity; f/s, frames/second

An example of how the adaptation of VCL intervals influenced the distribution of motile spermatozoa for the three swimming speed classes is presented in Fig. 1 for vervet monkey spermatozoa. It is clear from Table 4 and Fig. 1 that if selected VCL intervals are too low for a particular species (e.g., default values in our study), most spermatozoa would be classified as rapid swimming speed class (Fig. 1A). If the adapted VCL cut-offs presented in Table 5 were used to re-evaluate the same sperm population, however, a different and more representative distribution of spermatozoa across the three subpopulations become apparent (Fig.1B) compared to using default VCL intervals (Fig. 1A). It should be noted that the different colored tracks in Fig. 1 (red, green and blue) do not correspond directly to the rapid, medium and slow swimming speed classes. Nevertheless, because only the VCL intervals were adjusted before the captured tracks were re-analyzed (Fig. 1B), it does indicate how the change in VCL cut-offs influences the three sub-populations.

No significant differences were found among the sperm kinematic parameters of the Total Average, Rapid Default and Rapid Adjusted subpopulations for most species (Table 4). The only two exceptions were the rhesus and vervet monkeys for which the swimming speed parameters were significantly greater in the Rapid Adjusted subpopulation compared to the other two subpopulations. The small percentage of motile spermatozoa in the Medium Default and Slow Default subpopulations of all six species seemed to be an "artefact" after observing the extremely slow swimming speeds of spermatozoa in these two classes. If only the Rapid Adjusted, Medium Adjusted and Slow Adjusted subpopulations are considered, the sperm kinematic parameters were significantly different among these three subpopulations for most parameters $(p<0.05)$. Examples of sperm motility tracks and kinematic parameters of human spermatozoa grouped into the three adjusted subpopulations using the adapted VCL intervals are 
presented in Fig. 2. It is important to note the difference in scale of the three diagrams to understand the details of the tracks.

\section{Discussion}

A normal sperm population consists of a heterogeneous combination of spermatozoa that can be grouped into subpopulations according to differences in measurable characteristics. Sperm subpopulations likely represent spermatozoa in different physiological states (Abaigar et al. 2001), because only a small percentage of spermatozoa possess all the mechanisms required for synchronous cell functions during sperm transport, capacitation, hyperactivation, acrosome reaction and egg penetration (Holt and van Look 2004). Because each sperm subpopulation responds differently to changes in the environment or when exposed to unnatural conditions or treatments, these subpopulations should be assessed when predicting male fertility potential (Holt and van Look 2004).

During the past two decades, many reports have appeared that identified and quantified sperm subpopulations in mammals using flow cytometry and CASA (sperm motility and morphometry) (Harrison 1996, Thurston et al. 1999, Abaigar et al. 1999, 2001, Quintero-Moreno et al. 2003, 2007, Martinez-Pastor et al. 2005, Núñez-Martínez et al. 2006). CASA systems provide detailed measurements of several parameters for both the whole sperm population and individual spermatozoa (Holt and van Look 2004). CASA, therefore, is an essential tool for identifying and studying sperm subpopulations. Because CASA analysis yields a large number of related motility parameters, most of the studies cited above focused on finding a suitable statistical method (e.g., multivariate cluster analysis, regression analysis or principal component analysis) to select the motility parameters that best identified sperm subpopulations in an ejaculate. Several of these studies mentioned the importance of sperm velocity, VCL and VSL in particular, to define sperm subpopulations and their response to drug treatments (Martinez-Pastor et al. 2005, Núñez-Martínez et al. 2006). 

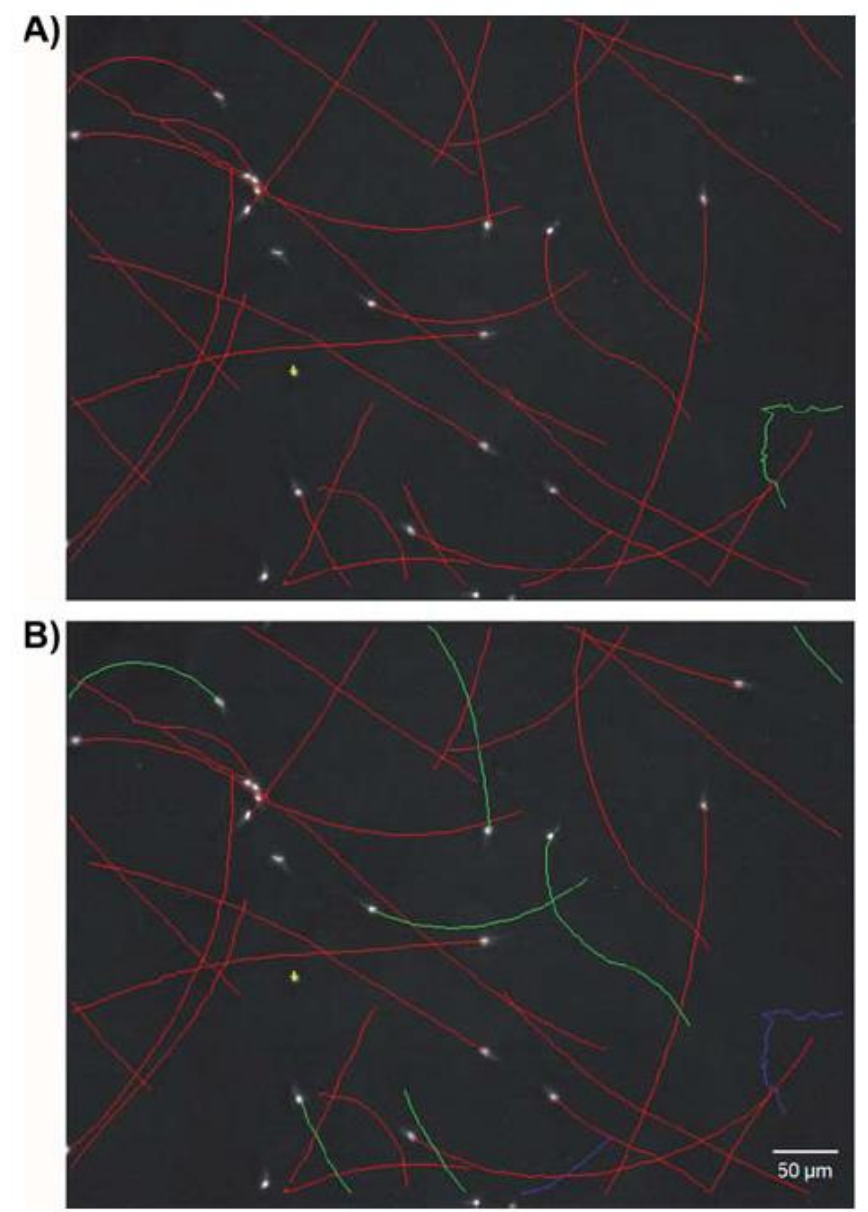

Fig. 1. Identical sperm motility tracks of vervet monkey spermatozoa illustrating how adaptations of VCL intervals for rapid, medium and slow swimming spermatozoa result in a change in the distribution of the three subpopulations captured at 50 frames/sec by the $\mathrm{SCA}^{\circledR}$ system. A) Default VCL cut-off values: rapid $>120<$ medium $>80<$ slow $>50$. B) Adjusted VCL cut-off values: rapid $>280<$ medium $>164<$ slow $>64$. The actual kinematic parameters for the spermatozoa in these three swimming speed classes are presented in Table 4 . Red tracks, rapid progressive swimming spermatozoa; green tracks, rapid swimming spermatozoa; blue tracks, medium progressive swimming spermatozoa; yellow crosses, static spermatozoa.

We used CASA-quantified sperm velocity to define sperm subpopulations in different mammalian species. In addition to measuring the percentage of total motile spermatozoa in a sperm population, most CASA systems also can measure alternative motility parameters that can be employed to assess the unique motility characteristics of sperm subpopulations. One such option, previously recommended by the World Health Organization (1999), is the type a-d classification of spermatozoa according to progression and speed (rapid or slow) of motile sperm. This classification system is based on human spermatozoa, however, and cannot be used for motility evaluations in other mammalian species.

The SCA ${ }^{\circledR}$ CASA system provides an additional option to identify and quantify sperm subpopulations by defining three motility classes: rapid, medium and slow according to the swimming speed of individual spermatozoa. Because grouping spermatozoa into 
one of these three motility classes is based on cut-off values for VCL, it is necessary to select the correct VCL intervals to classify individual spermatozoa. These cut-off values were determined by assessing sperm motility and swimming characteristics of a preselected motile sperm population for six mammalian species.

The high percentage of spermatozoa allocated to the default rapid swimming speed class was expected, because the techniques employed for sperm selection included mostly motile spermatozoa in the sperm populations evaluated. The low percentage spermatozoa allocated to the default medium and slow swimming speed classes, however, as well as their significantly lower sperm velocities, created a "false" picture of the motility characteristics of spermatozoa in these two swimming speed classes. It was evident from our results that the arbitrarily selected default VCL intervals for the three swimming speed classes were too low and too generalized for all six species evaluated.

Using the rhesus monkey as an example, the VCL cut-off values had to be adjusted to double that of the default VCL intervals to identify properly the three sperm subpopulations. 


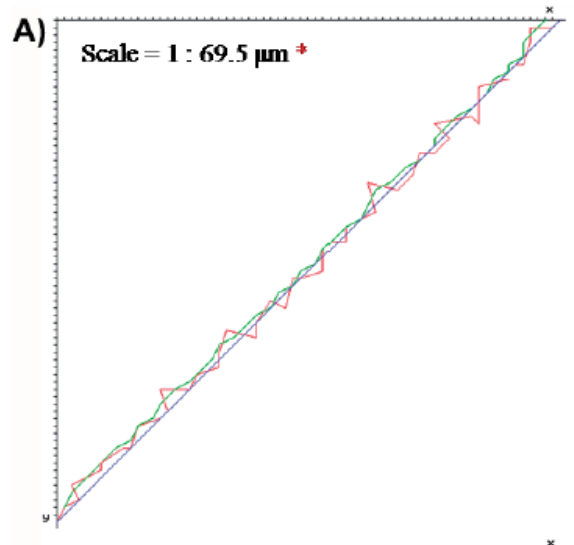

\begin{tabular}{|c|c|}
\hline Classification & Rapid \\
\hline VCL $(\mu \mathrm{m} / \mathrm{s})$ & 130.1 \\
\hline VSL ( $(\mu \mathrm{m} / \mathrm{s})$ & 98.3 \\
\hline $\operatorname{VAP}(\mu \mathrm{m} / \mathrm{s})$ & 102.9 \\
\hline IIN (\%) & 75.5 \\
\hline STR $(\%)$ & 95.5 \\
\hline wOB (\%) & 79.1 \\
\hline ALE(נm) & 2.2 \\
\hline BCF (Hz) & 27.0 \\
\hline Classification & Mediun \\
\hline VCL $(\mathrm{mm} / \mathrm{s})$ & 75.7 \\
\hline VSL $(\mu \mathrm{m} / \mathrm{s})$ & 46.9 \\
\hline VAP $(\mu \mathrm{m} / \mathrm{s})$ & 66.3 \\
\hline IIN (\%) & 62.0 \\
\hline $\operatorname{sTR}(\%)$ & 70.8 \\
\hline WOB (\%) & 87.5 \\
\hline ALH (um) & 1.7 \\
\hline BCF (Hz) & 15.3 \\
\hline Classification & Slow \\
\hline $\operatorname{VCL}(\mu \mathrm{m} / \mathrm{s})$ & 50.1 \\
\hline VSL ( $1 \mu \mathrm{m} / \mathrm{s})$ & 20.5 \\
\hline VAP $(\mu \mathrm{m} / \mathrm{s})$ & 34.4 \\
\hline I.IN (\%) & 40.9 \\
\hline STR (\%) & 59.5 \\
\hline WOB (\%) & 68.6 \\
\hline ALH(jm) & 1.0 \\
\hline $\mathrm{BCF}(\mathrm{Hz})$ & 7.7 \\
\hline
\end{tabular}

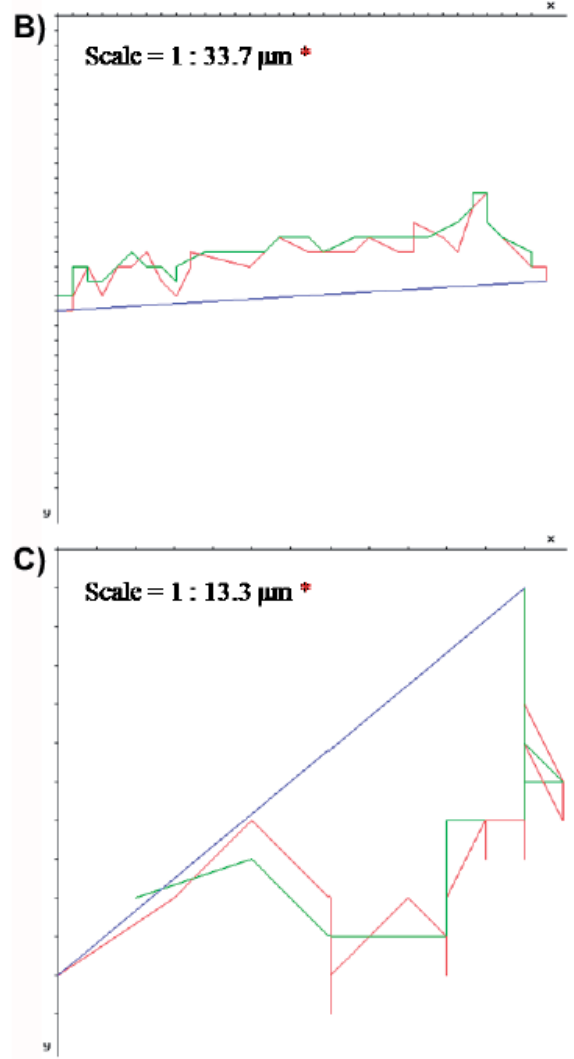

7.7

* Please note difference in scale for $\mathrm{A}, \mathrm{B}$ and $\mathrm{C}$

Fig. 2. Representative sperm motility tracks of sample of human spermatozoa illustrating differences in the kinematic parameters of the three swimming speed classes captured at 50 frames/sec by the $\mathrm{SCA}^{\circledR}$ system and after adjusting the VCL intervals. A) Rapid swimming sperm. B) Medium swimming sperm. C) Slow swimming sperm. Red line, VCL; green line, VAP; blue line, VSL. The average kinematic parameters of spermatozoa included in these three swimming speed classes are presented in Table 4. VCL, curvilinear velocity; VSL, straight-line velocity; VAP, average path velocity; LIN, linearity; STR, straightness; WOB, wobble; ALH, amplitude of lateral head displacement; BCF, beat cross frequency.

By adjusting the VCL intervals to classify 80,15 and $5 \%$ motile spermatozoa for each of the three swimming speed classes respectively, produced more reliable measurements of sperm subpopulations. This was confirmed by the fact that, although the kinematic measurements of the adjusted rapid swimming speed class were similar to those of the default rapid class $(p>0.05)$, the medium and slow swimming speed classes showed more convincing kinematic characteristics. Moreover, the adjusted VCL intervals permitted the establishment of unique kinematic characteristics for each swimming speed class $(p<0.05)$. These characteristics and the VCL intervals also were specific for each mammalian species evaluated. Although evaluation of sperm populations at different 
time intervals and frame rates had significant effects on VCL for some of the species evaluated (data not shown), no differences were found in the VCL cut-off values.

It should be noted that we focused on preselected motile sperm populations that included mainly spermatozoa exhibiting activated motility and that probably also had a high fertility potential (Windsor 1997, Larsen et al. 2000, Kasai et al. 2002, Marchetti et al. 2002). Thus, the VCL cut-off values that we determined are guidelines and should be used only to generate baseline data for various sperm motility parameters for future studies. When studying semen or sperm populations under different circumstances or experimental protocols, VCL intervals should be determined using an appropriate rationale for selecting the percentage of spermatozoa to be included in each swimming speed class (e.g., 80, 15 and 5\% in our study). In a clinical environment, semen from donors could be used to determine proper VCL intervals for the swimming speed classes and compared to semen or selected sperm from patients with infertility problems.

The value of our quantitative method for identifying different sperm subpopulations will become known after it is used to assess the effect of a specific treatment on each subpopulation. Because this technique defines subpopulations according to the percentage of total motility, we expect that the effect of any drug treatment will become evident by changes in the distribution (percentage) of spermatozoa among the three swimming speed classes. It should be stressed, however, that if too high or too low VCL intervals were selected, incorrect distribution of the defined sperm subpopulations could mask the effects of treatment.

We have studied the effects of different metabolic inhibitors on sperm motility and have shown that when employing the method to define sperm subpopulations described here, the inhibitory effect became apparent only when the percentage of rapid swimming spermatozoa were evaluated rather than the total percentage of motile spermatozoa (unpublished observations). Several previous studies have reported similar shifts in the distribution of spermatozoa across sperm subpopulations after drug treatment. For example, Abaigar et al. (1999) found that treatment with bicarbonate and caffeine caused a major shift of spermatozoa from the active, nonlinear movement subpopulation to a subpopulation that exhibited rapid, linear movement. The effects of short-term storage or cryo-preservation on sperm motility in gazelle, horse, red deer and dog has been investigated by assessing changes in the distribution of sperm subpopulations over time or after thawing (Abaigar et al. 2001, Quintero-Moreno et al. 2003, Martinez-Pastor et al. 2005, Núñez-Martínez et al. 2006).

One critique of our technique to define sperm subpopulations could be the use of only one CASA motility parameter to classify spermatozoa into different groups. Previous studies have considered all CASA parameters for defining such subpopulations. By employing various statistical methods, other investigators have selected only the motility parameters that revealed the most relevant information for identifying sperm subpopulations (Quintero-Moreno et al. 2003, 2007). It is evident from our results, however, that when spermatozoa were classified into three groups using only sperm velocity, the subpopulations in all six species differed significantly from each other for 
most of the kinematic parameters assessed. This clearly indicates that three unique sperm subpopulations were defined using our simplified technique. Martinez-Pastor et al. (2005) stated that using progressive or rapid sperm motility to define sperm subpopulations is not as informative as identification by cluster analysis. The same investigators, however, reported that individual CASA parameters still provided more useful information about the effect of cryopreservation on sperm subpopulations than when only mean values for motility were assessed. The advantage of using only sperm swimming speed to define sperm subpopulations is that these data can be extracted readily from the CASA analysis (Martinez-Pastor et al. 2005) after VCL cut-offs have been determined.

Our technique allowed identification of sperm subpopulations by using the swimming speed of individual spermatozoa. This quantitative method also provided objective measurements of the characteristics of each swimming speed class, but only if the VCL intervals were adjusted to species-specific cut-off values. Future studies should focus on whether evaluation of the percentages of rapid, medium and slow swimming spermatozoa and their kinematic parameters are more efficient for showing an effect on sperm motility than using only the percentage for total motility.

\section{Acknowledgments}

The authors are grateful to Ms Charon de Villiers and colleagues, MRC Delft Animal Centre; Dr. Jürgen Seier and colleagues, MRC Primate Unit; Prof. Stefan du Plessis and postgraduate students, Reproductive Research Laboratory, Division of Medical Physiology, Department of Biomedical Sciences, Stellenbosch University; Prof. Schalk Cloete; Ms Annelie Kruger and colleagues, Elsenburg Experimental Farm for their assistance during collection of semen samples. This study was supported by the National Research Foundation (NRF) and Senate Research Fund of the University of the Western Cape.

Declaration of interest: GvdH collaborates with Microptic SL (Barcelona, Spain), the company from which the $\mathrm{SCA}^{\circledR}$ system was purchased. For our research, the $\mathrm{SCA}^{\circledR}$ system was employed solely as a research tool to define sperm subpopulations. Any opinion, findings, conclusions and recommendations expressed in this article are those of the authors; the NRF accepts no liability with regard to this report. 


\section{References}

Abaigar T, Holt WV, Harrison RAP, del Barrio G (1999) Sperm subpopulations in boar (Sus scrofa) and gazelle (Gazella dama mhorr) semen as revealed by pattern analysis of computer-assisted motility assessments. Biol. Reprod. 60: 3241.

Abaigar T, Cano M, Pickard AR, Holt WV (2001) Use of computer-assisted sperm motility assessment and multivariate pattern analysis to characterize ejaculate quality in Mohor gazelles (Gazella dama mhorr): effects of body weight, electroejaculation technique and short-term semen storage. Reproduction 122: 265-273.

Agarwal A, Sharma RK, Nelson DR (2003) New semen quality scores developed by principal component analysis of semen characteristics. J. Androl. 24: 343-352.

Brinders J (1994) The Effects of Different Media and Gamma Irradiation on Quantitative Sperm Motility in the Wistar rat. MSc thesis. University of the Western Cape, Department of Physiological Sciences.

Christie B (2000) Doctors revise Declaration of Helsinki. BMJ 321: 913.

Cloete SWP, Gilmour AR, Olivier JJ, van Wyk JB (2004) Genetic and phenotypic trends and parameters in reproduction, greasy fleece weight and live weight in Merino lines divergently selected for multiple rearing ability. Aus. J. Exp. Agric. 44: 745-754.

de Villiers C, Seier JV (2010) Sudden death in chacma baboons (Papio ursinus). Lab. Anim. Eur. 10: 10-12.

Gomendio M, Malo AF, Garde J, Roldan ERS (2007) Sperm traits and male fertility in natural populations. Reproduction 134: 19-29.

Harrison RAP (1996) Capacitation mechanisms and the role of capacitation as seen in eutherian mammals. Reprod. Fertil. Dev. 8: 581-594.

Holt WV, van Look KJW (2004) Concepts in sperm heterogeneity, sperm selection and sperm competition as biological foundations for laboratory tests of semen quality. Reproduction 127: 527-535.

Humphries S, Evans JP, Simmons LW (2008) Sperm competition: linking form to function. BMC Evol. Biol. 8: 319.

Jørgensen N, Auger J, Giwercman A, Irvine DS, Jensen TK, Jouannet P, Keiding N, Le Bon C, MacDonald E, Pekuri AM, Scheike T, Simonsen M, Suominen J, Skakkeboek NE (1997) Semen analysis performed by different laboratory teams: an inter-variation study. Int. J. Androl. 20: 201-208.

Kasai T, Ogawa K, Mizuno K, Nagai S, Uchida Y, Ohta S, Fujie M, Suzuki K, Hirata S, Hoshi K (2002) Relationship between sperm mitochondrial membrane potential, sperm motility, and fertility potential. Asian J. Androl. 4: 97-103.

Kraemer M, Fillion C, Martin-Pont B, Auger J (1998) Factors influencing human sperm kinematic measurements by the Celltrak computer-assisted sperm analysis system. Hum. Reprod. 13: 611-619.

Larsen L, Scheicke T, Jensen TK, Bonde JP, Ernst E, Hjollund NH, Zhou Y, Skakkebak NE, Giwercman A (2000) Computer-assisted semen analysis parameters as predictors for fertility of men from the general population. Hum. Reprod. 15: 1562-1567. 
Mahadevan MM, Miller MM, Moutos DM (1997) Absence of glucose decreases human fertilization and sperm movement characteristics in vitro. Hum. Reprod. 12: $119-123$.

Malo AF, Garde JJ, Soler AJ, García AJ, Gomendio M, Roldan ERS (2005) Male fertility in natural populations of red deer is determined by sperm velocity and the proportion of normal spermatozoa. Biol. Reprod. 72: 822-829.

Marchetti C, Obert G, Deffosez A, Formstecher P, Marchetti P (2002) Study of mitochondrial membrane potential, reactive oxygen species, DNA fragmentation and cell viability by flow cytometry in humans. Hum. Reprod. 17: $1257-1265$.

Maree L, du Plessis SS, Menkveld R, van der Horst G (2010) Morphometric dimensions of the human sperm head depend on the staining method used. Hum. Reprod. 25: 1369-1382.

Martinez-Pastor F, Garcia-Macias V, Alvarez M, Herraez P, Anel L, de Paz P (2005) Sperm subpopulations in Iberian red deer epididymal sperm and their changes trough the cryopreservation process. Biol. Reprod. 72: 316-327.

Mocé E, Graham JK (2008) In vitro evaluation of sperm quality. Anim. Reprod. Sci. 105: 104-118.

Mortimer D (1994) Practical Laboratory Andrology. Oxford University Press, New York. pp. 135-157.

Mortimer ST (1997) A critical review of the physiological importance and analysis of sperm movement in mammals. Hum. Reprod. Update 3: 403-439.

Núñez-Martínez I, Moran JM, Peña FJ (2006) A three-step statistical procedure to identify sperm kinematic subpopulations in canine ejaculates: changes after cryopreservation. Reprod. Dom. Anim. 41: 408-415.

Parsons SDC, de Villiers C, Gey von Pittius NC, Warren RM, van Helden PD (2010) Detection of Myco-bacterium kansasii infection in a rhesus macaque (Macaca mulatta) using a modified QuantiFERON-TB Gold assay. Vet. Immunol. Immunopathol. 136: 330-334.

Quintero-Moreno A, Miró J, Rigau T, Rodríguez-Gil JE (2003) Identification of sperm subpopulations with specific motility characteristics in stallion ejaculates. Theriogenology 59: 1973-1990.

Quintero-Moreno A, Rigau T, Rodríguez-Gil JE (2004) Regression analysis and motile sperm subpopulation structure study as improving tools in boar semen quality analysis. Theriogenology 61: 673-690.

Quintero-Moreno A, Rigau T, Rodríguez-Gil JE (2007) Multivariate cluster analysis regression procedures as tools to identify motile sperm subpopulations in rabbit semen and to predict semen fertility and litter size. Reprod. Dom. Anim. 42: 312-319.

Rijsselaere T, van Soom A, Tanghe S, Coryn M, Maes D, de Kruif A (2005) New techniques for the assessment of canine semen quality: a review. Theriogenology 64: 706-719.

Seier JV (1986) Breeding vervet monkeys in a closed environment. J. Med. Primatol. 5: 339-349.

Sifer C, Sasportes T, Barraud V, Poncelet C, Rudant J, Porcher R, CedrinDurnerin I, Martin-Pont B, Hugues JN, Wolf JP (2005) World Health Organization grade 'a' motility and zona-binding test accurately predict IVF 
outcome for mild male factor and unexplained infertilities. Hum. Reprod. 20: 2769-2775.

Tayama K, Fujita H, Takahashi H, Nagasawa A, Yano N, Yuzawa K, Ogata A (2006) Measuring mouse sperm parameters using a particle counter and sperm quality analyzer: a simple and inexpensive method. Reprod. Toxicol. 22: 92-101.

Thurston LM, Watson PF, Holt WV (1999) Sources of variation in the morphological characteristics of sperm subpopulations assessed objectively by a novel automated sperm morphology analysis system. J. Reprod. Fertil. 117: 271280 .

Turner RM (2003) Tales from the tail: what do we really know about sperm motility? J. Androl. 24: 790-803.

van der Horst G, Maree $\mathbf{L}$ (2009) SpermBlue ${ }^{\circledR}$ : a new universal stain for human and animal sperm which is also amenable to sperm morphology analysis. Biotech. \& Histochem. 84: 299-308.

van der Horst G, Maree L, Kotze SH, O'Riain J (2011) Sperm structure and sperm motility in the eusocial naked mole rat, Heterocephalus glaber: a case of degenerative orthogenesis the absence of sperm competition? BMC Evol. Biol. 11: 351.

Windsor DP (1997) Mitochondrial function and ram sperm fertility. Reprod. Fertil. Dev. 9: 279-284.

World Health Organization (1999) WHO Laboratory Manual for the Examination of Human Semen and Sperm-Cervical Mucus Interaction, $4^{\text {th }}$ ed. Cambridge University Press, Cambridge, UK. pp. 1-138.

Zinaman MJ, Brown CC, Selevan SG, Clegg ED (2000) Semen quality and human fertility: a prospective study with healthy couples. J. Androl. 21: 145-153. 